\title{
Optimal Dynamic Formation Control of Multi-Agent Systems in Constrained Environments *
}

\author{
Xinmiao Sun ${ }^{\text {a }}$, Christos G. Cassandras ${ }^{\text {a }}$ \\ ${ }^{a}$ Division of Systems Engineering and Center for Information and Systems Engineering, Boston University, 15 Saint Mary's Street, \\ Brookline, MA, 02446, USA
}

\begin{abstract}
We address the optimal dynamic formation problem in mobile leader-follower networks where an optimal formation is generated to maximize a given objective function while continuously preserving connectivity. We show that in a convex mission space, the connectivity constraints can be satisfied by any feasible solution to a mixed integer nonlinear optimization problem (MINLP). For the class of optimal formation problems where the objective is to maximize coverage, we show that the optimal formation is a tree which can be efficiently constructed without solving a MINLP. In a mission space constrained by obstacles, we separate the formation process into intervals with no obstacles detected and intervals where one or more obstacles are detected. In the latter case, we propose a minimum-effort reconfiguration approach for the formation which still optimizes the objective function while avoiding the obstacles and ensuring connectivity. We include simulation results illustrating this dynamic formation process.
\end{abstract}

Key words: Formation control; Optimal coverage; Co-operative control; Optimization; Network connectivity preservation.

\section{Introduction}

The multi-agent system framework consists of a team of autonomous agents cooperating to carry out complex tasks within a given environment that is potentially highly dynamic, hazardous, and even adversarial. The overall objective of the system may be time-varying and combines exploration, data collection, and tracking to define a "mission", see Cao et al. (2013); Shamma (2008); Choi et al. (2009); Cassandras and Li (2005). In many cases, mobile agents are required to establish and maintain a certain spatial configuration, leading to a variety of formation control problems. These problems are generally approached in two ways: in the leader-follower setting, an agent is designated as a team leader moving on some given trajectory with the remaining agents tracking this trajectory while maintaining the formation; in the leaderless setting the formation must be maintained without any such benefit. Examples of formation control problems may be found in Yamaguchi and Arai (1994); Desai et al. (1999); Ji and Egerstedt (2007); Wang

* The authors' work is supported in part by NSF under grants CNS-1239021, ECCS-1509084, and IIP-1430145, by AFOSR under grant FA9550-15-1-0471, and by ONR under grant N0001409-1-1051. Corresponding author Christos G. Cassandras. Tel. (617)353-7154. Fax. (617)353-4830.

Email addresses: xmsun@bu.edu (Xinmiao Sun), cgc@bu.edu (Christos G. Cassandras). and Xin (2013) and references therein. In robotics, this is a well-studied problem; for instance in Yamaguchi and Arai (1994), a desired shape for a networked strongly connected group of robots is achieved by designing a quadratic spread potential field on a relative distance space. In Desai et al. (1999), a leader and several followers move in an area with obstacles which necessitate the transition from an initial formation shape to a desired new shape; however, the actual choice of formations for a particular mission is not addressed in Desai et al. (1999), an issue which is central to our approach in this paper. In Ji and Egerstedt (2007) the authors consider the problem of preserving connectivity when the nodes have limited sensing and communication ranges; this is accomplished through a control law based on the gradient of an edge-tension function. More recently, in Wang and Xin (2013), the goal is to integrate formation control with trajectory tracking and obstacle avoidance using an optimal control framework.

In this paper, we take a different viewpoint of formations. Since agent teams are typically assigned a mission, there is an objective (or cost) function associated with the team's operation which depends on the spatial configuration (formation) of the team. Therefore, we view a formation as the result of an optimization problem which the agent team solves in either centralized or distributed manner. We adopt a leader-follower approach, whereby the leader moves according to a trajectory that only he/she controls. During the 
mission, the formation is preserved or must adapt if the mission (hence the objective function) changes or if the composition of the team is altered (by additions or subtractions of agents) or if the team encounters obstacles which must be avoided. In the latter case in particular, we expect that the team adapts to a new formation which still seeks to optimize an objective function so as to continue the team's mission by attaining the best possible performance. The problem is complicated by the fact that such adaptation must take place in real time. Thus, if the optimization problem determining the optimal formation is computationally demanding, we must seek a fast and efficient control approach which yields possibly sub-optimal formations, but guarantees that the initial connectivity attained is preserved. Obviously, once obstacles are cleared, the team is expected to return to its nominal optimal formation.

Although the optimal dynamic formation control framework proposed here is not limited by the choice of tasks assigned to the team, we will focus on the dynamic coverage control problem because its static version is well studied and amenable to efficient distributed optimization methods; see Cortes et al. (2004); Cassandras and Li (2005); CaicedoNuez and Zefran (2008); Caicedo-Nunez and Zefran (2008); Breitenmoser et al. (2010); Zhong and Cassandras (2011), while also presenting the challenge of being generally nonconvex and sensitive to the agent locations during the execution of a mission. The local optimality issue, which depends on the choice of objective function, is addressed in Sun et al. (2014); Schwager et al. (2008); Gusrialdi et al. (2013), while the problem of connectivity preservation in view of limited communication ranges is considered in $\mathrm{Ji}$ and Egerstedt (2007); Zhong and Cassandras (2011).

The contribution of this paper is to formulate an optimization problem which jointly seeks to position agents in a twodimensional mission space so as to optimize a given objective function while at the same time ensuring that the leader and remaining agents maintain a connected graph dictated by minimum distances between agents, thus resulting in an optimal formation. The minimum distances may capture limited communication ranges as well as constraints such as maintaining desired relative proximity between agents. We show that the solution to this problem guarantees such connectivity. For the class of optimal coverage control problems, we show that an optimal formation is a tree whose construction is much more computationally efficient than that of a general connected graph. The formation becomes dynamic as soon as the leader starts moving along a trajectory which may either be known to all agents in advance or determined only by the leader. Thus, it is the team's responsibility to maintain an optimal formation. We show that this is relatively simple as long as no obstacles are encountered. When one or more obstacles are encountered (i.e., they come within the sensing range of one or more agents), then we propose a scheme for adapting with minimal effort to a sequence of new formations which maintain connectivity while still seeking to optimize the original team objective.
The paper is organized as follows. In Sec. II, we formulate a general optimal formation control problem and, for a convex feasible space, derive a mixed integer nonlinear optimization problem whose solution is shown to ensure connectivity while maintaining an optimal formation. In Sec. III, we focus on optimal coverage control problems, prove that a tree is an optimal formation, and propose an algorithm to construct such a tree in a convex mission space. In Sec. IV, we address the optimal formation problem in a mission space with obstacles. We propose an algorithm to first obtain a connected formation and then optimize it while maintaining connectivity. Simulation results are included in Sec. V.

\section{Optimal Formation Problem}

Consider a set of $N+1$ agents with a leader labeled 0 and $N$ followers labeled 1 through $N$ in a mission space $\Omega \in \mathbb{R}^{2}$. Agent $i$ is located at $s_{i}(t) \in \mathbb{R}^{2}$ and let $\mathbf{s}(t)=\left(s_{0}(t), \ldots, s_{N}(t)\right)$ be the full agent location vector at $t$. The leader follows a predefined trajectory $s_{0}(t)$ over $t \in[0, T]$ which is generally not known in advance by the remaining agents. We model the agent team as a directed graph $\mathscr{G}(\mathbf{s})=(\mathscr{N}, \mathscr{E}, \mathbf{s})$, where $\mathscr{N}=\{0,1, \ldots, N\}$ is the set of agent indices and let $\mathscr{N}_{F}=$ $\{1, \ldots, N\} \subset \mathscr{N}$ be the set of follower indices. In this model, the set of edges $\mathscr{E}=\{(i, j): i, j \in \mathscr{N}\}$ contains all possible agent pairs for which constraints may be imposed.

In performing a mission, let $H(\mathbf{s}(t))$ be an objective function dependent on the agent locations $\mathbf{s}(t)$. If the locations are unconstrained, the problem is posed as $\max _{\mathbf{s}(t) \in \Omega} H(\mathbf{s}(t))$ subject to dynamics that may characterize the motion of each agent. If $t$ is fixed, then this is a nonlinear parametric optimization problem over the mission space $\Omega$ (Zhong and Cassandras (2011)). If, in addition, agents are required to satisfy some constraints relative to each other's position, then a formation is defined as a graph that satisfies these constraints. We then introduce a Boolean variable $c\left(s_{i}, s_{j}\right)$ to indicate whether two agents satisfy these constraints:

$$
c\left(s_{i}, s_{j}\right)=\left\{\begin{array}{l}
1 \text { all constraints are satisfied } \\
0 \text { otherwise }
\end{array}\right.
$$

and if $c\left(s_{i}, s_{j}\right)=1$ we say that agents $i$ and $j$ are connected. A loop-free path from the leader to agent $i, \pi_{i}=$ $\{0, \ldots, a, b, \ldots, i\}$, is defined as an ordered set where neighboring agents are connected such that $c\left(s_{a}, s_{b}\right)=1$. Let $\Pi_{i}$ be the set of all possible paths from $i$ connected to the leader. The graph $\mathscr{G}(\mathbf{s})$ is connected if $\Pi_{i} \neq \emptyset$ for all $i \in \mathscr{N}_{F}$. We can now formulate an optimal formation problem with connectivity preservation as follows, for any fixed $t \in[0, T]$ :

$$
\begin{array}{cc} 
& \max _{\mathbf{s}(t) \in \Omega} H(\mathbf{s}(t)) \\
\text { s.t. } \quad & s_{i}(t) \in F \subseteq \Omega, \quad i \in \mathscr{N}_{F} \\
& s_{0}(t) \text { is given } \\
\mathscr{G}(\mathbf{s}(t)) \text { is connected }
\end{array}
$$




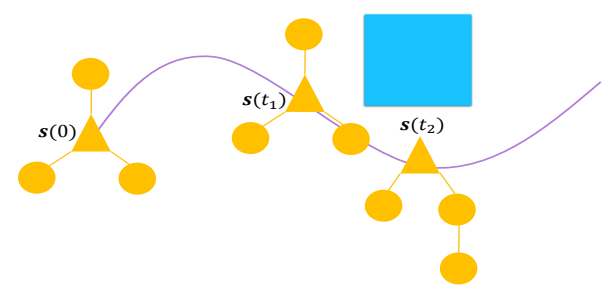

Fig. 1. A mission space example where the triangle is the leader and the purple line is a predefined trajectory. The circles are followers and the rectangle is an obstacle. The formation is maintained in $\left[0, t_{1}\right]$, but at $t_{2}$ a new formation is needed.

For the sake of generality, we impose the constraint $s_{i}(t) \in$ $F \subseteq \Omega$ for all follower agents to capture the possibility that a formation is constrained. The feasible space $F$ can be convex (e.g., followers may be required to be located on one side of the leader relative to a line in $\Omega$ that goes through $s_{0}(t)$ ) or non-convex (e.g., followers may be forbidden to enter polygonal regions, possibly physical obstacles, and $F$ is the set $\Omega$ excluding all interior points of these regions). The solution to this problem is an optimal formation at time $t$ and is denoted by $\mathscr{G}^{*}(\mathbf{s}(t))$. Given a time interval $\left[t_{1}, t_{2}\right]$, the formation is maintained in $\left[t_{1}, t_{2}\right]$ if $s_{i}(t)-s_{i}\left(t_{1}\right)=s_{0}(t)-s_{0}\left(t_{1}\right)$ holds for all $t \in\left[t_{1}, t_{2}\right], i \in \mathscr{N}_{F}$; otherwise, it is a new formation. Figure 1 shows an example of optimal dynamic formation control in a mission space with obstacles. Clearly, this is a challenging problem. To begin with, the last constraint in (2) is imprecise and may be different in a convex or non-convex feasible space. In addition, the computational complexity of obtaining a solution may be manageable in determining an initial formation but becomes infeasible if a new formation $\mathscr{G}^{*}(\mathbf{s}(t))$ is required during the real-time execution of a mission. We first propose a general approach to solve this problem in a convex feasible space for arbitrary $H(\mathbf{s}(t))$. In the next section, we will limit ourselves to the class of optimal coverage problems in both convex and nonconvex feasible spaces and show how to take advantage of the specific structure of $H(\mathbf{s}(t))$ in such cases.

In a convex feasible space, the simplest connection constraints are of the form $d_{i j}(t) \equiv\left\|s_{i}(t)-s_{j}(t)\right\| \leq C_{i j}$ for some pair $(i, j), i, j \in\{0,1, \ldots, N\}$, where $C_{i j}>0$ is a given scalar. This may be the minimum distance needed to establish communication or $d_{i j}$ may be used to enforce a specific desired geometric shape in the formation. Techniques based on the graph Laplacian are often used to solve this kind of problem, e.g., Olfati-Saber and Murray (2004); Merris (1994). However, our goal is to determine a formation which solves the optimization problem in (2) for a given $H(\mathbf{s}(t))$. Thus, we describe next an approach to transform the last constraint in (2) into a mixed integer nonlinear optimization problem by introducing a set of flow variables over $\mathscr{G}(\mathbf{s})$. The leader 0 is assumed to be a source node which sends $N$ units of flow through the graph $\mathscr{G}(\mathbf{s})$ to all other agents. Let $\rho_{i j} \in \mathbb{Z}^{+}, i \in \mathscr{N}, j \in \mathscr{N}_{F}$ be an integer flow amount through link $(i, j)$. Note that, in general, $\rho_{i j} \neq \rho_{j i}$ and that either $\rho_{i j}>0$ or $\rho_{j i}>0$ implies that $c\left(s_{i}, s_{j}\right)=1$. We can then de- fine a flow vector $\rho=\left(\rho_{01}, \rho_{11}, \ldots, \rho_{N 1}, \ldots, \rho_{0 N}, \ldots, \rho_{N N}\right)$. Observe that $\rho_{i 0}, i \in \mathscr{N}$ is not a flow variable in $\rho$ since the leader is not allowed to receive any flows from the followers. For each follower $j$, we define an auxiliary variable $N_{j}$ to be the net flow at node $j$ :

$$
N_{j}=\sum_{i \in \mathscr{N}} \rho_{i j}-\sum_{i \in \mathscr{N}_{F}} \rho_{j i}
$$

Using this notation, we introduce next a number of linear constraints that represent a connected graph. First, the leader provides $N$ units of flow:

$$
\sum_{i \in \mathscr{N}_{F}} \rho_{0 i}=N
$$

Next, each follower $j$ must receive a net flow $N_{j}=1$ in order to ensure that there is one path from the leader to $j$ :

$$
N_{j}=\sum_{i \in \mathscr{N}} \rho_{i j}-\sum_{i \in \mathscr{N}_{F}} \rho_{j i}=1, \quad j \in \mathscr{N}_{F}
$$

To prohibit self loops, we require that

$$
\rho_{i i}=0, \quad i \in \mathscr{N}_{\mathscr{F}}
$$

Finally, the maximal flow capacity is upper bounded by the source amount $N$ :

$$
\rho_{i j} \leq N, \quad i \in \mathscr{N}, \quad j \in \mathscr{N}_{F}
$$

Observe that (4) and (5) are linearly dependent since $\sum_{j} N_{j}=$ $N$. Thus, the constraint (4) is redundant and may be omitted.

Theorem 1 If there exists a flow vector $\rho$ such that constraints (5)-(7) hold, then there exists a connected graph $\mathscr{G}(\mathbf{s})$. Moreover, the number of possible graphs is finite.

\section{Proof: See Appendix.}

Observe that $\rho_{i j}>0$ indicates a connection between agents $i$ and $j$. This can be combined with the constraint $d_{i j}(t) \leq C_{i j}$ to write $\rho_{i j}\left(d_{i j}(t)-C_{i j}\right) \leq 0$ for all edges $(i, j)$ in $\mathscr{G}(\mathbf{s})$. Moreover, the convex set $F$ can be expressed through linear constraints. Thus, the optimal formation problem with connectivity preservation at any fixed $t \in[0, T]$ becomes a Mixed Integer Nonlinear Problem (MINLP):

$$
\begin{gathered}
\min _{\mathbf{s}(t), \rho}-H(\mathbf{s}(t), \rho) \\
\text { s.t. } \quad s_{i}(t) \in F \subseteq \Omega, \quad i=0, \ldots, N \\
\sum_{i \in \mathscr{N}} \rho_{i j}-\sum_{i \in \mathscr{N}_{F}} \rho_{j i}=1, \quad j \in \mathscr{N}_{F} \\
\rho_{i j}\left(d_{i j}(t)-C_{i j}\right) \leq 0, \quad i \in \mathscr{N}, \quad j \in \mathscr{N}_{F} \\
\rho_{i i}=0, \quad i \in \mathscr{N}_{F} \\
\rho_{i j} \leq N, \quad i \in \mathscr{N}, \quad j \in \mathscr{N}_{F}
\end{gathered}
$$


Note that any agent position vector $\mathbf{s}(t)$ specifies a graph at time $t$. The role of $\rho$ is to ensure that this graph is connected by satisfying the constraints in (8), thus creating an optimal formation. However, there is no advance information regarding what the optimal formation looks like and how the optimal formation changes over time as the leader moves in a time interval $[0, T]$ unless $H(\mathbf{s}(t))$ is given some specific structure. For the rest of this paper, we focus on a class of problems which impose a particular structure on $H(\mathbf{s}(t))$.

\section{Optimal coverage control problems}

Multi-agent systems involve interactions between agents and points in the mission space or between agents with each other. We are typically interested in maximizing rewards resulting from such interactions, thus leading to objective functions of the general form

$$
H(\mathbf{s}(t))=\int_{\Omega} R(x) P(x, \mathbf{s}(t)) d x
$$

where $R(x)$ describes some information associated with point $x \in \Omega$ (e.g., its value relative to other points in the mission space) and $P(x, \mathbf{s})$ is the reward resulting from the interaction between $x \in \Omega$ and agents located at $\mathbf{s}(t)=\left(s_{0}(t), \ldots, s_{N}(t)\right)$. The formulation of problems aiming to control $\mathbf{s}(t)$ so as to maximize $H(\mathbf{s}(t))$ in (9) is very general and includes, for instance, the class of consensus problems, which is relatively simple compared to the harder class (due to nonconvexity) of optimal coverage or persistent monitoring problems (see Zhong and Cassandras (2011); Schwager et al. (2011); Cassandras et al. (2013); Sakurama et al. (2015)). Agents are assumed to be equipped with some sensing and some communication capabilities. In particular, we assume that agent $i$ 's sensing is limited to a set $\Omega_{i}(t) \subset \Omega$. For simplicity, we let $\Omega_{i}(t)$ be a circle centered at $s_{i}(t)$ with radius $\delta_{i}$. Thus, $\Omega_{i}(t)=\left\{x: d_{i}(x, t) \leq \delta_{i}\right\}$ where $d_{i}(x, t)=\left\|x-s_{i}(t)\right\|$, the standard Euclidean norm. To further maintain simplicity (without affecting the generality of the analysis), we set $\delta_{i}=\delta$ for all agents.

In coverage problems, the function $R(x): \Omega \rightarrow \mathbb{R}$ captures an a priori estimate of the frequency of event occurrences at $x$ and is referred to as an "event density" satisfying $R(x) \geq 0$ for all $x \in \Omega$ and $\int_{\Omega} R(x) d x<\infty$. For the formation problems we consider here, we assume that the event density is a constant for any $x \in \Omega$. To define $P(x, \mathbf{s}(t))$ in (9), let $p_{i}\left(x, s_{i}(t)\right)$ be the probability that agent $i$ detects an event occurring at point $x$. This function is defined to have the following properties: $(i) p_{i}\left(x, s_{i}(t)\right)=0$ if $x \notin \Omega_{i}(t)$, and $(i i)$ $p_{i}\left(x, s_{i}(t)\right) \geq 0$ is a monotonically nonincreasing function of $d_{i}(x, t)$. The overall sensing detection probability is denoted by $\hat{p}_{i}\left(x, s_{i}(t)\right)$ and defined as

$$
\hat{p}_{i}\left(x, s_{i}(t)\right)=\left\{\begin{array}{lll}
p_{i}\left(x, s_{i}(t)\right) & \text { if } & x \in \Omega_{i}(t) \\
0 & \text { if } & x \notin \Omega_{i}(t)
\end{array}\right.
$$

Note that $\hat{p}_{i}\left(x, s_{i}(t)\right)$ may not be continuous in $s_{i}(t)$. We may now define the joint detection probability that an event at $x \in \Omega$ is detected by at least one of the $N$ cooperating nodes in the network:

$$
P(x, \mathbf{s}(t))=1-\prod_{i=0}^{N}\left[1-\hat{p}_{i}\left(x, s_{i}(t)\right)\right]
$$

where we assume that agents sense independently of each other. In addition to sensing, the communication capabilities of agents are defined by their relative distance: agents $i$ and $j$ can establish a communication link if $\left\|s_{i}(t)-s_{j}(t)\right\| \leq$ $C_{i j}$. For simplicity, $C_{i j}=C$ for all $i$ and $j$. Thus, in this class of problems a formation is required to maintain full communication among agents. Finally, one of the agents, indexed by 0 , is designated as the leader whose position $s_{0}(t)$ is given. We are interested in formations maximizing the total detection probability over the mission space $\Omega$, so that the objective in (8) is $H(\mathbf{s}(t), \rho)=\int_{\Omega} R(x) P(x, \mathbf{s}(t)) d x$ with $R(x), P(x, \mathbf{s}(t))$ as defined above and $\rho$ the flow vector defined in the previous section.

This MINLP is NP-hard (Köppe (2012)) and its solution is computationally costly, so that it is not realistic to expect resolving it over the course of a mission as the leader moves. In fact, it is not always necessary to repeatedly solve this problem over $[0, T]$. Theorem 2 presents a condition under which we only need to solve the problem at $t=0$. This simply formalizes the rather obvious fact that if no new constraints (e.g., obstacles) are encountered over $t \in(0, T]$, then the optimal formation at $t=0$ can be preserved by maintaining fixed relative positions for all agents.

Theorem 2 Assume that $R(x)=R$ for all $x \in F$. Let $\mathbf{s}(0)$ be an optimal solution of problem (8) at $t=0$ and that $s_{0}(t)$ is known to all followers for all $t \in(0, T]$. If $s_{i}(t)=$ $s_{i}(0)+s_{0}(t)-s_{0}(0), i \in \mathscr{N}_{F}$ and $\Omega_{i}(t) \subset F, i \in \mathscr{N}$, then $\mathbf{s}(t)$ maximizes $H(\mathbf{s}(t))$ in (9) with $P(x, \mathbf{s}(t))$ in (11).

\section{Proof: See Appendix.}

The implication of Theorem 2 is that when a mission space has no obstacles in it or the leader follows a trajectory where no obstacles are encountered by any agent, our problem is reduced to one of ensuring that all agents accurately track the leader's trajectory. We may discretize time so that agents update their locations at $0<t_{1}<\cdots<t_{K}=T$. Assuming that problem (8) is solved at $t=0$, an optimal formation is obtained and we subsequently strive to maintain this formation until a significant "event" occurs such as an agent failure, a change in objective function $H(\mathbf{s}(t))$, or encountering obstacles; at such a point, some amount of reconfiguration is required while still aiming to maximize $H(\mathbf{s}(t))$.

Figures 2 and 3 show two examples of optimal formation obtained by solving (8) at time $t$ with $s_{0}(t)$ located at the center of the mission space. To get Fig. 3, we simply add constraints $s_{i x} \leq s_{0 x}$ in (8) and the methodology remains the 
same. Moreover, any such linear constraint can be accommodated. In these examples, the optimal formation graphs $\mathscr{G}^{*}(\mathbf{s})$ are trees (The definition of a tree can be found in Bertsimas and Tsitsiklis (1997)). Clearly, if we know that an optimal formation is a tree, the problem is much simpler to solve. We will show next that this is indeed the case for formations constructed to solve optimal coverage problems.

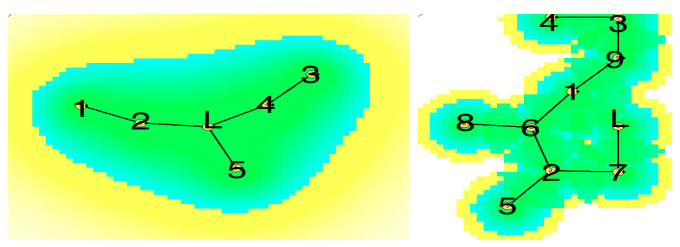

Fig. 2. Optimal formation for Fig. 3. Optimal formation for 9 5 followers and a leader in a followers and a leader. Followbounded mission space. ers are constrained to the left of the leader.

Theorem 3 Assume that the feasible space is $F=\Omega=\mathbb{R}^{2}$, $C<2 \delta, R(x)=R$ for all $x \in F$ and $p_{i}\left(x, s_{i}(t)\right)=p\left(x, s_{i}(t)\right)$. Then, an optimal formation $\mathscr{G}^{*}(\mathbf{s})(t)$ for problem (8) with the objective function (9) with $P(x, \mathbf{s}(t))$ in (11) is a tree with the distance between connected agents given by $C$.

Proof: See Appendix, which includes three Lemmas required for the proof.

This theorem enables us to construct an optimal solution rather than solving the MINLP problem, thus dramatically decreasing computation. Algorithm 1, which is of complexity $O\left(N^{2}\right)$, is an example of a simple procedure through which such a tree can be constructed.

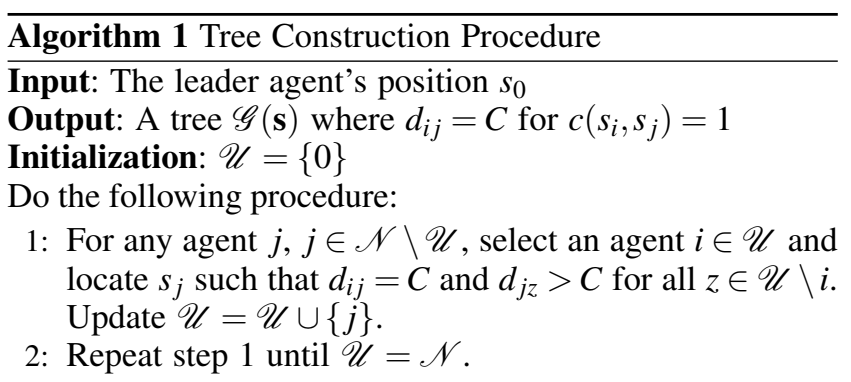

Note that the feasible space $F$ is assumed to be $\mathbb{R}^{2}$ in Theorem 3. This implies that a tree is optimal as long as no agent's position is limited by a constraint such as an obstacle or the finite boundaries of the mission space. To address this issue, we define a union coverage area set by $A_{u}(\mathbf{s})=\cup_{i=0}^{N} \Omega_{i}$. Then $A_{u}(\mathbf{s}) \subset \mathbb{R}^{2}$ due to the fact that the sensing range of any agent is limited (see the definition of $\Omega_{i}$ ). A tree solution, say $\mathscr{G}(\mathbf{s})$ obtained by Algorithm 1 may be globally optimal if $A_{u}(\mathbf{s}) \subset F$ or infeasible otherwise. For the latter case, we can obtain a local optimum by the following gradient-based algorithm

$$
s_{i}^{k+1}=s_{i}^{k}+\zeta_{k} \frac{\partial H(\mathbf{s})}{\partial s_{i}^{k}}, \quad k=0,1, \ldots
$$

where the step size sequence $\left\{\zeta_{k}\right\}$ is selected (e.g., Bertsekas (1995)) to ensure convergence and the calculation of $\frac{\partial H(\mathbf{s})}{\partial s_{i}^{k}}$ can be found in Zhong and Cassandras (2011).

\section{Optimal Dynamic Formation Control in a Mission Space with Obstacles}

We have thus far solved an optimal dynamic formation problem with connectivity constraints in a convex feasible space $F$ by solving a MINLP or simply constructing a tree when Theorem 3 applies. In the latter case, we can construct a solution with minimal computation, as in Algorithm 1. However, this method may fail when $F$ is non-convex, e.g., when $F$ cannot be described through linear or nonlinear constraints. In this section, we address the optimal dynamic formation problem in a mission space with obstacles, thus considering a non-convex feasible space.

We model the obstacles as $m$ non-self-intersecting polygons denoted by $M_{j}, j=1, \ldots, m$. The interior of $M_{j}$ is denoted by $\stackrel{\circ}{M}_{j}$, so that the overall feasible space is $F=\Omega \backslash\left(\stackrel{\circ}{M}_{1} \cup\right.$ $\left.\ldots \cup M_{m}\right)$, i.e., the space $\Omega$ excluding all interior points of the obstacles. In this setting, we seek to ensure the following two requirements. First, the distance between two connected agents must be $\leq C$. We define $c_{1}\left(s_{i}, s_{j}\right)$ to indicate whether this requirement is satisfied:

$$
c_{1}\left(s_{i}, s_{j}\right)=\left\{\begin{array}{l}
1\left\|s_{i}-s_{j}\right\| \leq C \\
0 \text { otherwise }
\end{array}\right.
$$

Second, the connected agents are required to have a line of sight with respect to each other. We define $c_{2}\left(s_{i}, s_{j}\right)$ to indicate this requirement:

$$
c_{2}\left(s_{i}, s_{j}\right)=\left\{\begin{array}{l}
1 \alpha s_{i}+(1-\alpha) s_{j} \in F \text { for all } \alpha \in[0,1] \\
0 \text { otherwise. }
\end{array}\right.
$$

Agents $i$ and $j$ satisfying $c_{1}\left(s_{i}, s_{j}\right)=1$ as well as $c_{2}\left(s_{i}, s_{j}\right)=$ 1 are referred to as connected. We also define $c\left(s_{i}, s_{j}\right)=$ $c_{1}\left(s_{i}, s_{j}\right) c_{2}\left(s_{i}, s_{j}\right)$.

A version of this connectivity preservation problem was addressed in Zhong and Cassandras (2011), where agents are required to remain connected with a fixed base while at the same time maximizing the objective function in (9). A gradient-based algorithm, termed Connectivity Preservation Algorithm (CPA), was developed for agent position updating and it was shown that, given an initially connected network and if only one agent updates its position at any given 


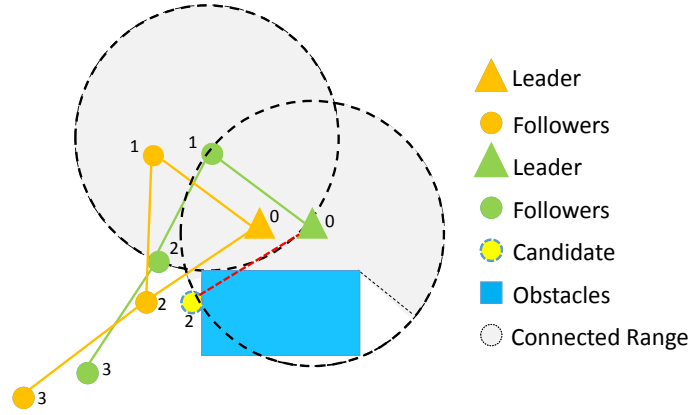

Fig. 4. An example of a connected network at $t$ and constructed connected network by Algorithm 2 at $t+\varepsilon$.

time, the CPA preserves connectivity. The algorithm is applied iteratively over one agent at a time and it converges to a (generally local) optimum. The CPA exploits the existence of distributed optimization algorithms for optimal coverage to attain optimal agent locations while also preserving connectivity to a base (details on the CPA and its complexity are provided in Zhong and Cassandras (2011)).

Our approach here is to take advantage of the CPA. In our problem, however, the conditions for applying the CPA do not generally hold; this is because the leader's motion does not take connectivity with its neighbors into account and the presence of an obstacle, for example, may cause it to disconnect from one or more followers. This is illustrated in Fig. 4: At time $t$, the agent network shown (represented by three orange circles and an orange triangle as the leader) is connected. At $t+\varepsilon$, the leader (triangle) moves to $s_{0}(t+$ $\varepsilon$ ) and if agent 2 moves to the point shown in yellow (as expected by Theorem 2), then it becomes disconnected from the leader because of the obstacle present. We propose an algorithm next to construct a connected graph, which may no longer be optimal in the sense of problem (8) but it does provide a valid initial condition for invoking the CPA described above (this is illustrated in Fig. 4 as the solid green graph). This immediately allows us to iteratively apply the CPA so as to obtain a new (locally optimal) formation.

Clearly, it is also possible to invoke (8) as soon as a formation reconfiguration is needed. However, the set $F$ is no longer convex and the computational complexity of this problem makes it infeasible for the on-line adaptation required, whereas the approach we propose and the use of the CPA render this process computationally manageable. In particular, whereas the MINLP is generally NP hard, in the CPA each agent $i$ determines its new position through a gradient-based scheme using only its neighbor set and its downstream and upstream agent sets relative to the leader (formally defined in the next section). When the number of agents increases, note that the the number of neighbors of $i$ may not be affected. The overall increase in complexity is linear in the network size.

Before proceeding, we identify the precise instants when formation reconfiguration is necessary due to obstacles en- countered by agents as the mission unfolds over $[0, T]$. We define two states that the agent team can be in: $(i)$ The constrained state occurs when the sensing capability of an agent is hindered by an obstacle, captured by the condition $\left(\bigcup_{i=0}^{N} \Omega_{i}\right) \cap\left(\bigcup_{i=1}^{m} \stackrel{\circ}{M}_{i}\right) \neq \emptyset$, and (ii) The free state corresponding to $\left(\bigcup_{i=0}^{N} \Omega_{i}\right) \cap\left(\bigcup_{i=1}^{m} \dot{M}_{i}\right)=\emptyset$. Thus, the interval $[0, T]$ is partitioned into free and constrained intervals with transitions at times $t_{f}^{0}<t_{c}^{1}<t_{f}^{1}<\ldots<t_{c}^{i}<t_{f}^{i}<\ldots t_{f}^{z}<$ $T$. When the agent network enters a free state at time $t_{f}^{k}$, $k=0, \ldots, z$, since $\left(\bigcup_{i=0}^{N} \Omega_{i}(t)\right) \cap\left(\bigcup_{i=1}^{m} \stackrel{\circ}{M}_{i}\right)=\emptyset$ for all $t \in$ $\left[t_{f}^{k}, t_{c}^{k+1}\right)$ and $F=\Omega \backslash\left(\stackrel{\circ}{1}_{1} \cup \ldots \cup \dot{M}_{m}\right)$, so $\Omega_{i}(t) \in F$ for any $i$ over $t \in\left[t_{f}^{k}, t_{c}^{k+1}\right)$, the optimal formation is maintained based on Theorem 2. Next, we consider how to generate optimal formations in constrained states.

Given a connected graph $\mathscr{G}(\mathbf{s})$, we have defined a loop-free path connecting agent $i$ to the leader as $\pi_{i}=\{0, \ldots, a, b, \ldots, i\}$; we have also defined $\Pi_{i}$ to be the set of all possible paths connecting $i$ to the leader. Let $\pi_{i, k}$ be the $k$ th path in $\Pi_{i}$ and we use $\pi_{i, k}^{j}$ to denote the $j$ th element in $\pi_{i, k}$. Let $\mathscr{D}_{i}=\cup_{j, k} w_{i}\left(\pi_{j, k}\right)$ be the set of agents downstream from $i$ (further away from the leader 0 ) where

$$
w_{i}\left(\pi_{j, k}\right)=\left\{\begin{array}{cl}
\pi_{j, k}^{l+1} & \text { if } i \in \pi_{j, k}, i \neq j \text { and } i=\pi_{j, k}^{l} \\
\emptyset & \text { otherwise }
\end{array}\right.
$$

We also define the set of upstream agents from $i$ as $\mathscr{U}_{i}=$ $\left\{j: i \in \mathscr{D}_{j}, j \in 0, \ldots, N\right\}$. The length of a path $\pi_{i, k}$ is defined as $\Psi\left(\pi_{i, k}\right)=\sum_{l=1}^{\left|\pi_{i, k}\right|-1}\left\|s_{\pi_{i, k}^{l}}-s_{\pi_{i, k}^{l+1}}\right\|$, where $\left|\pi_{i, k}\right|$ is the cardinality of $\pi_{i, k}$. For agent $i$, the shortest path connected to the leader is

$$
\pi_{i}^{*}=\arg \min _{\pi_{i, k} \in \Pi_{i}} \Psi\left(\pi_{i, k}\right)
$$

For example in Fig. 4, in path $\pi_{3,1}=\{0,2,3\}$ we have 3 $\in \mathscr{D}_{2}, 0 \in \mathscr{U}_{2}, \Psi\left(\pi_{3,1}\right)=\left\|s_{0}-s_{2}\right\|+\left\|s_{2}-s_{3}\right\|$; for path $\pi_{3,2}=\{0,1,2,3\}$, we have $\Psi\left(\pi_{3,2}\right)=\left\|s_{0}-s_{1}\right\|+\left\|s_{1}-s_{2}\right\|+$ $\left\|s_{2}-s_{3}\right\|$. Therefore, $\pi_{3}^{*}=\pi_{3,1}$ is the shortest path from agent 3 to the leader.

Let $\pi_{i}$ and $\pi_{j}$ be two paths. Then, we define $\pi_{i}+\pi_{j}=$ $\left\{\pi_{i}, \pi_{k}\right\}$, where $\pi_{k}=\pi_{j} \backslash \pi_{i}$, as an ordered set. Note that $\pi_{i}+\pi_{j}$ is generally different from $\pi_{j}+\pi_{i}$ because of the order involved. Given a connected graph $\mathscr{G}(\mathbf{s})$, We define

$$
Q(\mathscr{G}(\mathbf{s}))=\pi_{1}^{*}+\ldots+\pi_{N}^{*}
$$

to be an ordered set containing a permutation of the agent set $\{0,1, \ldots, N\}$ constructed so as to start with the shortest path $\pi_{1}^{*}$ from 0 to agent 1 , followed by $\pi_{2}^{*} \backslash \pi_{1}^{*}$ and so on. It immediately follows from this construction that the first element of $Q(\mathscr{G}(\mathbf{s}))$ is 0 and that $|Q(\mathscr{G}(\mathbf{s}))|=N+1$. Therefore, we can rewrite $Q(\mathscr{G}(\mathbf{s}))$ as

$$
Q(\mathscr{G}(\mathbf{s}))=\left\{0, q_{2}, \ldots, q_{N+1}\right\}
$$


where $q_{j} \in \mathscr{N}_{F}, j=2, \ldots, N+1$. For example, in Fig. 4, at time $t, Q(\mathscr{G}(\mathbf{s}(t)))=\{0,1,2,3\}$. We show next that $Q(\mathscr{G}(\mathbf{s}))$ has the following property regarding the order of its elements.

Lemma 4 If $q_{i}$ is the $i$ th element of $Q(\mathscr{G}(\mathbf{s}))$ constructed from a connected graph $\mathscr{G}(\mathbf{s})$, then there exists $q_{j} \in \mathscr{U}_{q_{i}}$ such that $q_{j}$ is the $j$ th element of $Q(\mathscr{G}(\mathbf{s}))$, and $j<i$ for all $q_{i} \in \mathscr{N}_{F}$.

Proof: If for all $q_{j} \in \mathscr{U}_{q_{i}}, j>i$, we cannot find a subset of $Q(\mathscr{G}(\mathbf{s}))$ that includes $\left\{q_{j}, q_{i}\right\}, q_{j} \in \mathscr{U}_{q_{i}}$, then there is no path connected to $q_{i}$. This contradicts the assumption that $Q(\mathscr{G}(\mathbf{s}))$ is constructed from a connected graph.

We also define a projection of $x \in \mathbb{R}^{2}$ on a set $A \in \mathbb{R}^{2}$ as

$$
P_{A}(x)=\arg \min _{y \in A}\|x-y\|
$$

Next, let $\mathscr{Y}\left(s_{i}\right)=\left\{y: y \in \mathbb{R}^{2}, c\left(s_{i}, y\right)=1\right)$. Recalling the definition of $c(\cdot, \cdot), \mathscr{Y}\left(s_{i}\right)$ is the set of points with which $s_{i}$ can establish a connection. For any subset of agents $\mathscr{V} \subset$ $\mathscr{N}$, let $\Sigma(\mathscr{V})=\bigcup_{i \in \mathscr{V}} \mathscr{Y}\left(s_{i}\right)$ be the union of all connection regions for agents in $\mathscr{V}$. For example, in Fig. 4, the grey area is $\Sigma(\mathscr{V})$ for $\mathscr{V}=\{0,1\}$ at time $t+\varepsilon$.

We are now ready to deal with the situation where the formation is in a constrained state and may lose connectivity at time $t+\varepsilon$ given that the graph $\mathscr{G}(\mathbf{s}(t))$ is connected. In particular, suppose that when the leader is about to move to $s_{0}(t+\varepsilon)$ and informs the followers, at least one of the agents will lose connectivity with the formation. Our task is to obtain an optimal formation at $t+\varepsilon$ and this is accomplished in two steps: $(i)$ Construct a connected graph $\mathscr{G}(\mathbf{s}(t+\varepsilon))$ for time $t+\varepsilon$, and $(i i)$ Use this connected graph $\mathscr{G}(\mathbf{s}(t+\varepsilon))$ as an input to invoke the CPA. Step $(i)$ is crucial because of the fact that the CPA relies on an initially connected graph before it can be executed to seek (locally) optimal agent locations which still preserve connectivity. This first step is carried out by constructing a connected graph through Algorithm 2. We use $\Delta_{L}(t)=s_{0}(t+\varepsilon)-s_{0}(t)$ to denote the

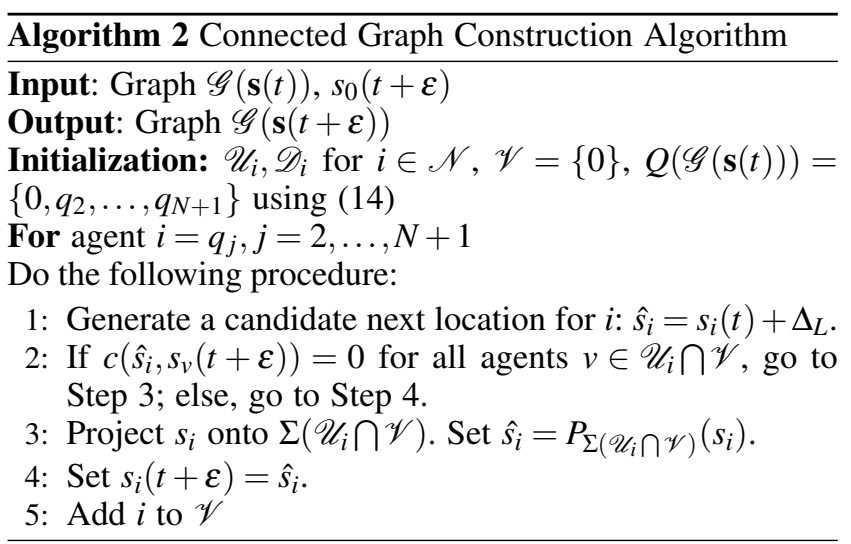

position change vector of the leader from $t$ to $t+\varepsilon$, where we assume that followers have the $\Delta_{L}(t)$ information available at $t$.

Theorem $4 \mathscr{G}(\mathbf{s}(t+\varepsilon))$ obtained by Algorithm 2 is connected.

Proof: See Appendix.

Obviously, Algorithm 2 does not provide a unique way to construct a connected graph. For example, the formation could be adjusted to a line or a star configuration with $s_{0}(t+$ $\varepsilon)$ as the center of the star. However, this would entail a major formation restructuring whereas in Algorithm 2 we seek to retain the closest possible formation to the original (optimal) one by setting candidate locations as seen in Step 1. If such a candidate is not feasible, then the agent will move a minimal distance (in the projection sense) to be connected.

Once step $(i)$ above is completed by obtaining this connected graph $\mathscr{G}(\mathbf{s}(t))$, step $(i i)$ is performed by invoking the CPA to optimize the agent locations within the new formation. Clearly, once obstacles are cleared and the agent team reenters a free state, we may revert to the original optimal formation. As for the complexity of this algorithm, in order to initialize $Q(\mathscr{G}(\mathbf{s}(t)))$, the time complexity for finding the shortest path is $O\left(N^{2}\right)$. In addition, to make a projection, agents need $O\left(N^{2}\right)$ comparisons, while the complexity of the CPA algorithm is $O(N)$. Therefore, the overall complexity is $O\left(N^{2}\right)$ in this case.

\section{Simulation Results}

In this section, we provide a simulation example illustrating what the optimal formation maximizing coverage in a mission space with obstacles looks like and how it changes at some significant instants (a video of the implementation of our optimal formation control approach for a team of small mobile robots in a laboratory setting can be found at http://www.bu.edu/codes/research/distributed-control/.)

We choose the event density functions to be uniform, i.e., $R(x)=1$. The mission space is a $60 \times 50$ rectangle. The distance constraint is $C=10$ and the sensing range of each agent is $\delta=8$. At every step, the leader moves to the right one distance unit per unit of time. The mission space is colored from dark to lighter as the joint detection probability decreases (the joint detection probability is $\geq 0.50$ for green areas, and near zero for white areas). The leader (labeled "L") moves along a predefined trajectory (the purple dashed line). There are 8 followers, indicated by numbers, which are restricted to locations on the left side of the leader during any movement.

Figures 5-10 show snapshots of the process at selected events of interest over $[0, T]$. Figure 5 shows the initial configuration at $t=0$, where the agent team is located in a convex feasible space. As shown in Sec. III, in this case, the optimal formation can be obtained by solving a MINLP. In the 
results shown, we have used TOMLAB, a MATLAB-based optimization solver. For the non-convex objective function defined in (9), the solution is usually a local maximum; we sought to find the best local (possibly global) optimum possible by implementing a multi-start algorithm on the solver. This is done at the start of the mission, when an off-line computationally intensive procedure is possible. Moreover, this local maximum can be improved by applying the CPA; in fact, in this example the use of the CPA led to an improvement from $H(\mathbf{s})=741.5$ to $H(\mathbf{s})=816.7$, as shown in Fig. 6. Thus, in general, supplying the CPA with an initial connected graph obtained by solving the MINLP enables it to converge to a better value. For example, Fig. 12 is a local maximum attained by starting with a star-like connected graph shown in Fig. 11 with the objective function value $H(\mathbf{s})=781.1$ (although this is still worse than the value in Fig. 6). In the time interval $[0,5]$, the formation is main-
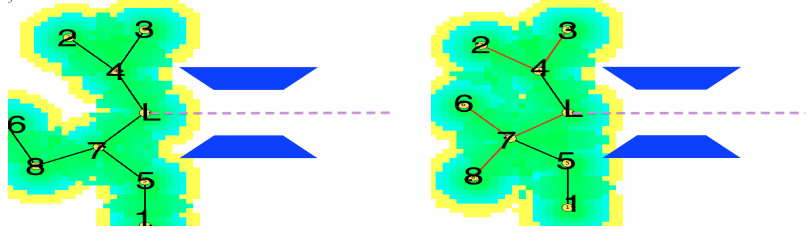

Fig. 5. At $t=0$, an optimal for- Fig. 6. The optimal formation mation is obtained by MINLP in Fig. 5 is improved by CPA. with $H(\mathbf{s})=741.5$

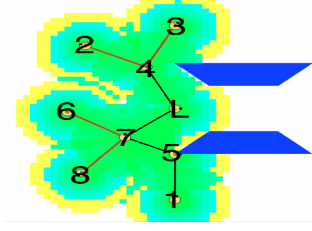

$H(\mathbf{s})=816.7$

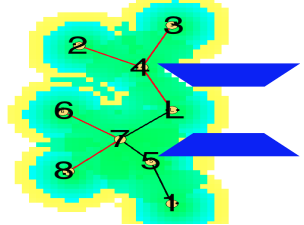

Fig. 7. At $t=5$, agent 5 needs Fig. 8. At $t=6$, agent 5 makes projection in Step 3 of Algo. 2 projection and CPA applies to Fig. 7
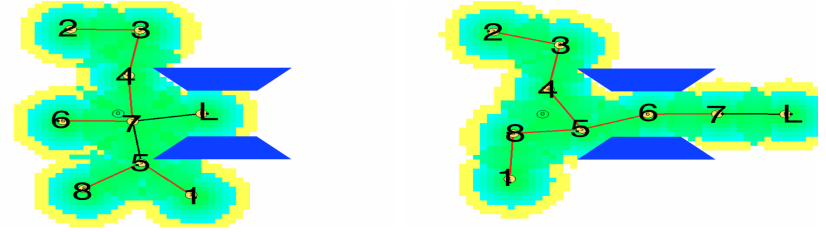

Fig. 9. At $t=12$, structure of the Fig. 10. At $t=35$, the end of the optimal formation changes mission
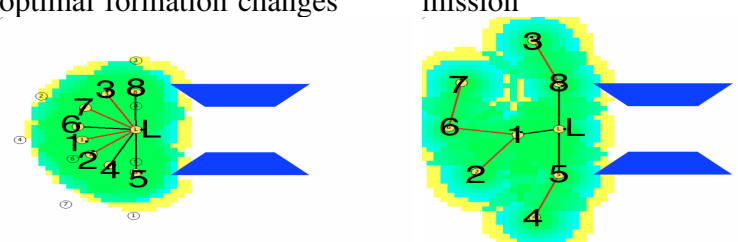

Fig. 11. A star-like connected Fig. 12. Apply CPA to Fig. 11. graph $H(\mathbf{s})=781.1$

tained. At $t=5$, agent 5 is located at a vertex of an obstacle and will therefore lose connectivity as the leader moves to the next step at $t=6$. At this point, agent 5 will determine its next position $s_{5}(6)$ by applying a projection at Step 3 of Algorithm 2. Note that only agent 5 needs to perform this projection, rather than the whole team of agents, hence the computational effort is minimal. Figure 8 captures the optimal formation following Fig. 7.

Observe that over the period $[0,12)$, although the optimal formation remains a tree, it is no longer the same as the original one. However, for each agent $i$, its downstream node set $\mathscr{D}_{i}$ and upstream node set $\mathscr{U}_{i}$ remain unchanged. At $t=12$, clearly, the structure of the formation has been changed. This is a consequence of either the projection step in Algorithm 2 or the CPA. At the end of the mission at $t=35$, the formation is shown in Fig. 10. The agents seek to form a line to go through the narrow region of the mission space while at the same time maximizing coverage. During the remaining interval $[12,35]$, the process is similar to what is seen over $[5,12]$.

As we pointed out in the last section, constructing a connected graph can be accomplished in a variety of ways. As shown in Fig. 11, a star-like graph is an inferior formation to that of Fig. 6; this is expected since the latter was obtained specifically to maximize the objective function in (9). In addition, a reconfiguration process as shown in Fig. 12 requires agents to move longer distances, hence consuming more energy.

\section{Conclusions and future work}

We have addressed the issue of optimal dynamic formation of multi-agent systems in mission spaces with constraints. When the agent team is in a free state (no obstacles in the mission space affecting them), a locally optimal solution of a MINLP can provide an initial formation that agents maintain or it is a good initial point for using the CPA (developed in prior work Zhong and Cassandras (2011)) to obtain a better local optimum. When the feasible space is non-convex and connectivity is lost, we have developed an algorithm to construct a connected graph as an input for the CPA while seeking to maintain the original formation with minimal effort. We have also shown that for the class of optimal formation problems where the objective is to maximize coverage, the optimal formation is (under certain conditions) a tree which can be efficiently constructed without solving a MINLP.

An interesting direction for future research is to deal with moving obstacles, assuming that agents have a model for the obstacle dynamics Schouwenaars et al. (2001). Moreover, we would like to investigate optimal dynamic formation control for more general classes of objective functions, beyond the coverage control problem.

\section{A Proof of Theorem 1}

In what follows, the binary operator + is used to concatenate two paths into a new path: If $\pi_{a}=\left\{\pi_{a}^{1}, \ldots, \pi_{a}^{m}\right\}$ and $\pi_{b}=$ $\left\{\pi_{b}^{1}, \ldots, \pi_{b}^{n}\right\}$, then $\pi_{a}+\pi_{b}=\left\{\pi_{a}^{1}, \ldots, \pi_{a}^{m}, \pi_{b}^{1}, \ldots, \pi_{b}^{n}\right\}$. 
We use a contradiction argument. Assume that at least one follower is not connected to the leader. Then the followers are divided into two sets: $N_{1}=\left\{k: \Pi_{k} \neq \emptyset\right\}$, which can connect to the leader and $N_{2}=\left\{j: \Pi_{j}=\emptyset\right\}$, which are not. Then $\rho_{k j}=0$ must be true for all $k \in N_{1}$ and $j \in N_{2}$. This is because if $\rho_{k j}>0$, then there exists a path $\pi_{j}=\left\{\pi_{k}\right\}+\{j\}$ where $\pi_{k} \in \Pi_{k}$, which contradicts the fact that $j \in N_{2}$. In addition, obviously $\rho_{0 j}=0$ for $j \in N_{2}$. Summing the lefthand-sides of all constraints (5) for $j \in N_{2}$, we obtain

$$
\begin{aligned}
& \sum_{j \in N_{2}} N_{j}=\sum_{j \in N_{2}}\left(\sum_{k \in \mathscr{N}} \rho_{k j}-\sum_{k \in N_{F}} \rho_{j k}\right) \\
= & \sum_{j \in N_{2}}\left[\sum_{k \in N_{1}} \rho_{k j}+\sum_{k \in N_{2}} \rho_{k j}+\rho_{0 j}-\left(\sum_{k \in N_{1}} \rho_{j k}+\sum_{k \in N_{2}} \rho_{j k}\right)\right] \\
= & -\sum_{j \in N_{2}} \sum_{k \in N_{1}} \rho_{j k} \leq 0
\end{aligned}
$$

Next, summing the right-hand-sides of the constraints (5) over $j \in N_{2}$ we get $\sum_{j \in N_{2}} N_{j}=\left|N_{2}\right| \geq 1$, contradicting (A.1). Therefore, the assumption is wrong and the graph $\mathscr{G}(\mathbf{s})$ is connected. The additional constraints (6)-(7) are necessary to ensure that the number of feasible flow vectors $\rho$ is finite. Clearly, (6) prohibits self-loops while (7) prevents an infinite number of solutions where edges $(i, j)$ in $\mathscr{G}(\mathbf{s})$ may take any unbounded flow value $\rho_{i j}>0$.

\section{B Proof of Theorem 2}

Let us introduce a local polar coordinate system for each agent $i$, so that the origin of $i$ 's such system is $s_{i}$ and the axes are parallel to those in the mission Cartesian coordinate system. Given any point $x=\left(x_{x}, x_{y}\right) \in F$, let $l=\left(r_{i}, \theta_{i}\right)$ be the polar coordinates in $i$ 's local coordinate system. Then, the transformation that maps $\left(r_{i}, \theta_{i}\right)$ onto the global coordinate system is $x=s_{i}(t)+\left[r_{i} \cos \theta_{i} r_{i} \sin \theta_{i}\right]^{T}$. Upon switching to this local coordinate system, the sensing probability becomes $p_{i}\left(x, s_{i}(t)\right)=p_{i}\left(r_{i}\right)$ if $r_{i}<\delta$. Since $\Omega_{i}(t) \subset F$ for all $t \in[0, T]$, the local sensing range of $s_{i}(t)$, which is denoted by $\Omega_{i}^{L}=\left\{\left(r_{i}, \theta_{i}\right): r_{i} \leq \delta, 0 \leq \theta_{i} \leq 2 \pi\right\}$, is time-invariant. Therefore, recalling (11), the objective function in (9) is

$$
\begin{aligned}
H(\mathbf{s}(t)) & =\int_{\Omega} R(x) P(x, \mathbf{s}(t)) d x=R \int_{\bigcup_{i=0}^{N} \Omega_{i}(t)} P(x, \mathbf{s}(t)) d x \\
& =R \int_{\bigcup_{i=0}^{N} \Omega_{i}^{L}} r_{i}\left\{1-\prod_{i=0}^{N}\left[1-p_{i}\left(r_{i}\right)\right]\right\} d r_{i} d \theta_{i}
\end{aligned}
$$

so that the objective function value remains fixed for any $t \in[0, T]$. Since for any agents $i$ and $j$, by assumption, $s_{i}(t)-$ $s_{j}(t)=s_{i}(0)+s_{0}(t)-s_{0}(0)-\left(s_{j}(0)+s_{0}(t)-s_{0}(0)\right)=$ $s_{i}(0)-s_{j}(0)$, and $\mathbf{s}(0)$ is an optimal solution of (8), it follows that $\mathscr{G}(\mathbf{s}(0))$ is connected, therefore, $\mathscr{G}(\mathbf{s}(t))$ is also connected and we conclude that $\mathbf{s}(t)$ maximizes $H(\mathbf{s}(t))$.

\section{Proof of Theorem 3}

Before we prove Theorem 3, we provide some notation and prove three lemmas. For simplicity, we omit $t$ from $s_{i}(t)$ in what follows. For any $i \in \mathscr{N}$, we define the maximal detection quality

$$
M_{i}=R \int_{\Omega_{i}} p_{i}\left(x, s_{i}\right) d x
$$

Lemma 1 If $p_{i}\left(x, s_{i}\right)=p\left(x, s_{i}\right)$ for all $i \in \mathscr{N}$, then $M_{i}=M$ for all $i \in \mathscr{N}$.

Proof: In the local polar coordinate system, $M_{i}$ is given by

$$
\begin{aligned}
M_{i} & =R \int_{\Omega_{i}^{L}} r_{i} p_{i}\left(r_{i}\right) d \theta_{i} d r_{i}=R \int_{0}^{2 \pi} \int_{0}^{C} r_{i} p_{i}\left(r_{i}\right) d \theta_{i} d r_{i} \\
& =2 \pi R \int_{0}^{C} r_{i} p_{i}\left(r_{i}\right) d r_{i}
\end{aligned}
$$

where $p_{i}\left(r_{i}\right)=p\left(r_{i}\right)$ if $p_{i}\left(x, s_{i}\right)=p\left(x, s_{i}\right)$, so the statement holds and $M_{i}$ is spatially invariant.

Lemma 2 Assume that $(i)$ the feasible space is $F=\Omega=\mathbb{R}^{2}$, $C \leq 2 \delta$ and $R(x)=R$ for all $x \in F$, and $(i i) p_{i}\left(x, s_{i}\right)=p\left(x, s_{i}\right)$ for all $i \in \mathscr{N}$. Then, the global optimal solution to problem (8) when $N=1$ is any position vector $\left(s_{0}, s_{1}\right)$ such that $\left\|s_{0}-s_{1}\right\|=C$ and the flow variable is $\rho=(1,0)$.

Proof: Recalling the sensing model (10) and the assumption $p_{i}\left(x, s_{i}\right)=p\left(x, s_{i}\right)$, the objective function in (9) for $N=1$ is

$$
\begin{aligned}
H(\mathbf{s}) & =\int_{F} R(x) P(x, \mathbf{s}) d x \\
& =R \int_{\Omega_{0}} p\left(x, s_{0}\right) d x+R \int_{\Omega_{1}} p\left(x, s_{1}\right) d x \\
& -R \int_{\Omega_{0} \cap \Omega_{1}} p\left(x, s_{0}\right) p\left(x, s_{1}\right) d x
\end{aligned}
$$

where the first two terms are the constants $M_{0}=M_{1}=M$ by Lemma 1 and we define a function $M_{2}\left(s_{i}, s_{j}\right)$ to represent the third term:

$$
M_{2}\left(s_{i}, s_{j}\right)=R \int_{\Omega_{i}\left(s_{i}\right) \cap \Omega_{j}\left(s_{j}\right)} p\left(x, s_{i}\right) p\left(x, s_{j}\right) d x
$$

Using $M$ and $M_{2}\left(s_{i}, s_{j}\right)$ in (C.3), we get

$$
H(\mathbf{s})=2 M-M_{2}\left(s_{0}, s_{1}\right)
$$

Let $\mathbf{s}_{C}=\left(s_{0}, s_{C}\right)$ and $\mathbf{s}_{z}=\left(s_{0}, s_{z}\right)$ be two feasible solutions where $\left\|s_{0}-s_{C}\right\|=C$ and $\left\|s_{0}-s_{z}\right\|=z, 0 \leq z<C$. We will show that $H\left(\mathbf{s}_{C}\right)$ is a global optimal solution, i.e., $H\left(\mathbf{s}_{C}\right)>$ $H\left(\mathbf{s}_{z}\right)$ holds for any $z$. To facilitate this proof, we establish a Cartesian coordinate system where $s_{0}=(0,0), s_{C}=(C, 0)$ and $s_{z}=(z, 0)$, as shown in Fig. C.1. Accordingly, we define the sensing range intersections $A_{a}=\Omega_{0}\left(s_{0}\right) \cap \Omega_{1}\left(s_{C}\right)$ and 


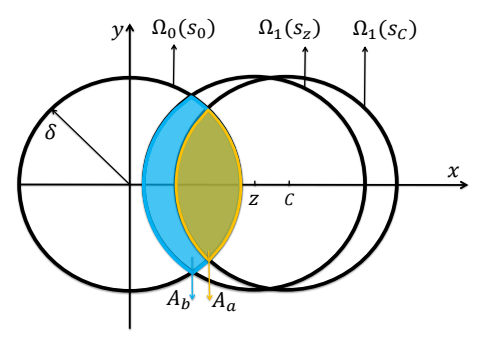

Fig. C.1. The sensing ranges of agents 0 and 1 where $s_{C}=(C, 0)$, $s_{z}=(z, 0)$ and $z \geq 2 \delta-C$.

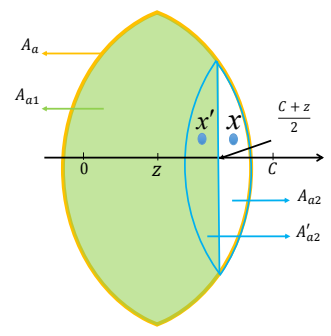

Fig. C.2. Subsets of $A_{a}$ when $z<2 \delta-C$. $A_{a}=A_{a 1} \cup A_{a 2}$ where $A_{a 1}$ is the green shape. $A_{a 2}^{\prime}$ (the blue-line green-filled shape) and $A_{a 2}$ (the blue-line write-filled shape) are symmetric with $x=(C+z) / 2$.

$A_{b}=\Omega_{0}\left(s_{0}\right) \cap \Omega_{1}\left(s_{z}\right)$. Note that $A_{a} \subset A_{b}$ due to the fact that $z<C$. It follows from (C.5) that

$$
\begin{aligned}
& H\left(\mathbf{s}_{C}\right)-H\left(\mathbf{s}_{z}\right) \\
= & R \int_{A_{b}} p\left(x, s_{0}\right) p\left(x, s_{z}\right) d x-R \int_{A_{a}} p\left(x, s_{0}\right) p\left(x, s_{C}\right) d x \\
= & R \int_{A_{a}} p\left(x, s_{0}\right)\left[p\left(x, s_{z}\right)-p\left(x, s_{C}\right)\right] d x \\
+ & R \int_{A_{b} \backslash A_{a}} p\left(x, s_{0}\right) p\left(x, s_{z}\right) d x
\end{aligned}
$$

Observing that if $z>C, \mathbf{s}_{z}$ is an infeasible solution, we will prove next that $H\left(\mathbf{s}_{C}\right)-H\left(\mathbf{s}_{z}\right)>0$ for any $0 \leq z<C$. Define a function $\tilde{p}\left(x, s_{z}, s_{C}\right)=p\left(x, s_{z}\right)-p\left(x, s_{C}\right)$ and observe that it has the following properties which are direct consequences of the monotonicity of the function $p(\cdot)$ in $\left\|x-s_{i}\right\|, i=C, z$ :

$P 1: \tilde{p}\left(x, s_{z}, s_{C}\right)\left\{\begin{array}{l}>0 \text { if } x_{x}<(z+C) / 2 \\ =0 \text { if } x_{x}=(z+C) / 2 \\ <0 \text { if } x_{x}>(z+C) / 2\end{array}\right.$

$P 2: \tilde{p}\left(x, s_{z}, s_{C}\right)=-\tilde{p}\left(x^{\prime}, s_{z}, s_{C}\right)$ if $\left\|x-s_{z}\right\|=\left\|x^{\prime}-s_{C}\right\|$ and $\left\|x-s_{C}\right\|=\left\|x^{\prime}-s_{z}\right\|$.

We then consider two cases: $z \geq 2 \delta-C$ and $0 \leq z<2 \delta-C$, corresponding to Fig. C.1 and Fig. C.2, respectively.

(1) If $z \geq 2 \delta-C$, then for any point $x \in A_{a}$ we have $x_{x}<(z+C) / 2$. Using $P 1$, we get $\tilde{p}\left(x, s_{z}, s_{C}\right)>0$. It follows that $H\left(\mathbf{s}_{C}\right)-H\left(\mathbf{s}_{z}\right)$ in (C.6) is positive since all integrands are positive:

$$
\begin{aligned}
H\left(\mathbf{s}_{C}\right)-H\left(\mathbf{s}_{z}\right) & =R \int_{A_{a}} p\left(x, s_{0}\right) \tilde{p}\left(x, s_{z}, s_{C}\right) d x \\
& +R \int_{A_{b} \backslash A_{a}} p_{0}\left(x, s_{0}\right) p_{1}\left(x, s_{z}\right) d x>0
\end{aligned}
$$

(2) If $0 \leq z<2 \delta-C$, then we divide the set $A_{a}$ into two subsets $A_{a 1}=\left\{x \mid x_{x} \leq(z+C) / 2, x \in A_{a}\right\}$ and $A_{a 2}=$ $\left\{x \mid x_{x}>(z+C) / 2, x \in A_{a}\right\}$. In the set $A_{a 1}$, we can find a subset $A_{a 2}^{\prime}=\left\{\left(z+C-x_{x}, x_{y}\right),\left(x_{x}, x_{y}\right) \in A_{a 2}\right\}$ which is symmetric to $A_{a 2}$ around an axis through $x_{x}=(z+C) / 2$ (see Fig. C.2). Then, for any point $x \in A_{a 2}$, there exists a point $x^{\prime} \in A_{a 2}^{\prime}$ such that $\left\|x-s_{z}\right\|=\left\|x^{\prime}-s_{C}\right\|$ and $\left\|x-s_{C}\right\|=\left\|x^{\prime}-s_{z}\right\|$. Using $P 2$, we obtain $\tilde{p}\left(x, s_{z}, s_{C}\right)=$ $-\tilde{p}\left(x^{\prime}, s_{z}, s_{C}\right)$. Hence,

$$
\int_{A_{a 2} \cup A_{a 2}^{\prime}} p\left(x, s_{0}\right) \tilde{p}\left(x, s_{z}, s_{C}\right) d x=0
$$

Let $A_{a 1}^{1}=A_{a 1} \backslash A_{a 2}^{\prime}$, therefore $A_{a}=A_{a 2} \cup A_{a 1}^{1} \cup A_{a 2}^{\prime}$. Accordingly, (C.6) is positive

$$
\begin{aligned}
& H\left(\mathbf{s}_{C}\right)-H\left(\mathbf{s}_{z}\right)=R \int_{A_{a 2} \cup A_{a 2}^{\prime}} p\left(x, s_{0}\right) \tilde{p}\left(x, s_{z}, s_{C}\right) d x \\
+ & R \int_{A_{a 1}^{1}} p\left(x, s_{0}\right) \tilde{p}\left(x, s_{z}, s_{C}\right) d x \\
+ & R \int_{A_{b} \backslash A_{a}} p\left(x, s_{0}\right) p\left(x, s_{z}\right) d x>0
\end{aligned}
$$

since the first term is zero due to (C.8), the second term is positive by $P 1$ and the third term is positive because of the positive integrand. Thus, in both cases (C.7) and (C.9) yield $H\left(\mathbf{s}_{C}\right)>H\left(\mathbf{s}_{z}\right)$, i.e., any vector $\left(s_{0}, s_{1}\right)$ such that $\left\|s_{0}-s_{1}\right\|=$ $C$ is the global optimal solution.

Lemma 2 establishes the fact that if there are only two agents in the feasible space, the optimal solution is obtained when the two agents are located at a distance of $C$ from each other. Using the definition of $A_{a}$, we define $M_{C}$ as

$$
M_{C}=R \int_{A_{a}} p\left(x, s_{0}\right) p\left(x, s_{C}\right) d x
$$

and obtain a final lemma:

Lemma 3 For agents $i$ and $j$, if $\left\|s_{i}-s_{j}\right\|=C$, then $M_{2}\left(s_{i}, s_{j}\right)=M_{C}$.

Proof: We establish a Cartesian coordinate system where the original point is $s_{i}$, the $x$-axis is in the same line as $s_{j}-s_{i}$, as shown in Fig. C.3. Then, it immediately follows that the result of the integration for $M_{2}\left(s_{i}, s_{j}\right)=M_{C}$.

Proof of Theorem 3: We prove the result by induction and the use of Lemmas 1-3. When $N=1$, by Lemma 2, the 


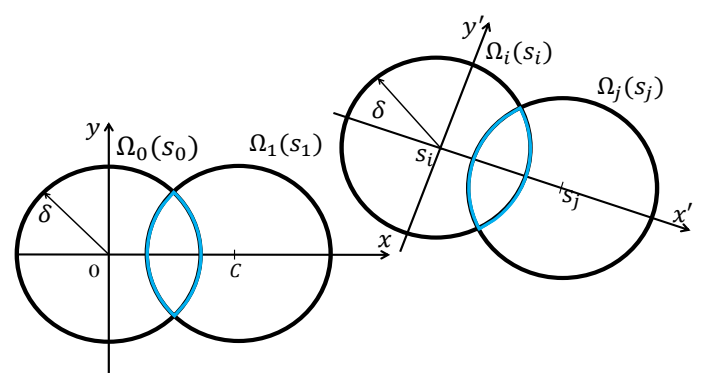

Fig. C.3. Two Cartesian coordinate systems $x-y$ and $x^{\prime}-y^{\prime}$

optimal formation is obtained by connecting the two agents with the distance between them being $C$. Next, we assume that when $N=k$ the optimal formation $\mathscr{G}_{k}(\mathbf{s})$ is a tree with the distance between the connected agents being $C$. Without loss of generality, these $k$ agents are labeled $1, \ldots, k$.

As $\mathscr{G}_{k}(\mathbf{s})$ is a tree, $|\mathscr{E}|=k-1$, i.e., there are $k-1$ pairs of connected agents. Let $\Omega\left(\mathscr{E}_{i}\right)=\Omega_{a_{i}} \cap \Omega_{b_{i}}$ where $\left(a_{i}, b_{i}\right)=$ $\mathscr{E}_{i} \in \mathscr{E}, i=1, \ldots, k-1$. In addition, it is impossible for more than two agents to be connected to each other because there is no cycle in the tree, which implies that $\Omega_{i} \cap \ldots \cap \Omega_{i} p=\emptyset$ for any $i^{p} \in\{0, \ldots k\}$ and $p>2$.

When $N=k+1$, the optimal formation $\mathscr{G}_{k+1}(\mathbf{s})$ is obtained by connecting the new agent to $\mathscr{G}_{k}(\mathbf{s})$ with the distance between connected agents being $C$. Assume that the new agent is labeled $h=k+1$. Agent $h$ may establish connection with $p(p \geq 1)$ agents at the same time (examples are shown in Figs. C.4-C.5). Accordingly, we denote the position of agent $h$ as $s_{h}^{p}$ if it connects to $p$ agents and let $\mathbf{s}_{p}=\left(s_{0}, \ldots, s_{k}, s_{h}^{p}\right)$. Next, we will show that $H\left(\mathbf{s}_{1}\right)>H\left(\mathbf{s}_{p}\right)$ for any $p \geq 1$.
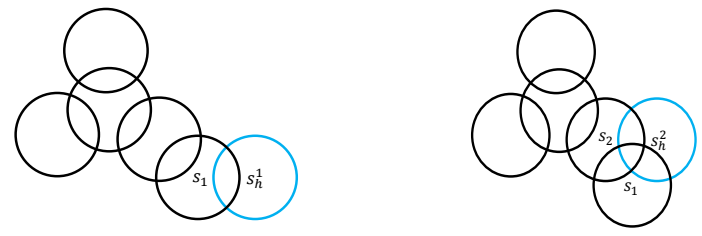

Fig. C.4. Agent $h$ connected to Fig. C.5. Agent $h$ connected to agent 1 agents 1 and 2

Similar to $M_{2}\left(s_{i}, s_{j}\right)$, we define a function

$$
M_{3}\left(s_{i}, s_{j}, s_{h}\right)=R \int_{\Omega\left(\mathscr{E}_{i}\right) \cap \Omega_{h}} p_{i}\left(x, s_{a_{i}}\right) p_{j}\left(x, s_{b_{i}}\right) p_{h}\left(x, s_{h}\right)
$$

and we can write the objective function $H\left(\mathbf{s}_{p}\right)$ for $N+1$ agents as follows:

$$
\begin{aligned}
H\left(\mathbf{s}_{p}\right) & =(k+2) M-\sum_{m, n \neq h} M_{2}\left(s_{m}, s_{n}\right) \\
& -\sum_{i=1}^{p} M_{2}\left(s_{i}, s_{h}^{p}\right)+\sum_{i=1}^{p-1} M_{3}\left(s_{a_{i}}, s_{b_{i}}, s_{h}^{p}\right)
\end{aligned}
$$

where $\left(a_{i}, b_{i}\right)=\mathscr{E}_{i}$. For $p=1,2, H\left(\mathbf{s}_{1}\right)$ and $H\left(\mathbf{s}_{2}\right)$ are

$$
\begin{aligned}
H\left(\mathbf{s}_{1}\right) & =(k+2) M-\sum_{m, n \neq h} M_{2}\left(s_{m}, s_{n}\right)-M_{2}\left(s_{1}, s_{h}^{1}\right) . \\
H\left(\mathbf{s}_{2}\right) & =(k+2) M-\sum_{m, n \neq h} M_{2}\left(s_{m}, s_{n}\right)-M_{2}\left(s_{1}, s_{h}^{2}\right)-M_{2}\left(s_{2}, s_{h}^{2}\right) \\
& +M_{3}\left(s_{a_{i}}, s_{b_{i}}, s_{h}^{2}\right)
\end{aligned}
$$

In Figs. C.4-C.5, $a_{1}=1$ and $a_{2}=2$. Note that $M_{2}\left(s_{1}, s_{h}^{1}\right)=$ $M_{2}\left(s_{1}, s_{h}^{2}\right)=M_{C}$ due to the fact that $\left\|s_{1}-s_{h}^{1}\right\|=\left\|s_{1}-s_{h}^{2}\right\|=C$ and invoking Lemma 3. Therefore,

$$
\begin{aligned}
& H\left(\mathbf{s}_{1}\right)-H\left(\mathbf{s}_{2}\right)=M_{2}\left(s_{2}, s_{h}^{2}\right)-M_{3}\left(s_{1}, s_{2}, s_{h}^{2}\right) \\
& =R \int_{\Omega_{1} \cap \Omega_{2} \cap \Omega_{h}} p_{1}\left(x, s_{i}\right) p_{h}\left(x, s_{h}^{2}\right)\left[1-p_{2}\left(x, s_{j}\right)\right] d x \\
& +R \int_{\Omega_{1} \cap \Omega_{h} \backslash \Omega_{1} \cap \Omega_{2} \cap \Omega_{h}} p_{1}\left(x, s_{1}\right) p_{h}\left(x, s_{h}^{2}\right) d x>0
\end{aligned}
$$

since both integrands are positive. Next, we obtain $H\left(\mathbf{s}_{1}\right)-$ $H\left(\mathbf{s}_{p}\right)$ :

$$
\begin{aligned}
& H\left(\mathbf{s}_{1}\right)-H\left(\mathbf{s}_{p}\right) \\
= & \sum_{i=1}^{p-1}\left(M_{2}\left(s_{i+1}, s_{h}^{p}\right)-M_{3}\left(s_{a_{i}}, s_{b_{i}}, s_{h}^{p}\right)\right) \\
= & (p-1)\left(H\left(\mathbf{s}_{1}\right)-H\left(\mathbf{s}_{2}\right)\right)
\end{aligned}
$$

This is true due to the fact that $\left\|s_{i}-s_{h}^{p}\right\|=C$ for $i=1, \ldots, p-1$, thus $M_{2}\left(s_{i+1}, s_{h}^{p}\right)=M_{C}$ using Lemma 3, and $M_{3}\left(s_{a_{i}}, s_{b_{i}}, s_{h}^{p}\right)=M_{3}\left(s_{1}, s_{2}, s_{h}^{p}\right)$.

We conclude that if agent $h=k+1$ is connected to $p$ agents of the tree, then $H\left(\mathbf{s}_{1}\right)-H\left(\mathbf{s}_{p}\right)=(p-1)\left(H\left(\mathbf{s}_{1}\right)-H\left(\mathbf{s}_{2}\right)\right)>$ 0 . In other words, the optimal solution is obtained when the newly added agent is connected to a single agent and the resulting formation $\mathscr{G}_{k+1}(\mathbf{s})$ is still a tree. Moreover, the distance between agent $h$ and the agent it is connected to, say $j$, is $C$, which can be proved with the same argument as that used in Lemma 2, i.e., we can perturb $s_{h}$ from $d_{j h}=C$ to $d_{j h}<C$ and show that $d_{j h}=C$ is the optimal solution. In addition, by Lemma 3 , connecting $h$ to any feasible agent $j$ results in the same objective function value.

Corollary For an optimal formation with $N+1$ agents, the objective function is $H(\mathbf{s})=(N+1) M-N M_{C}$ where $M$ and $M_{C}$ are as defined in Lemma 1 and (C.10).

\section{Proof of Theorem 4}

Since $\mathscr{G}(\mathbf{s}(t))$ is connected, $\mathscr{U}_{i} \neq \emptyset$ for $i \in \mathscr{N}_{F}$. We then use induction to prove that the graph constructed by agents in $\mathscr{V}$ remains connected at Step 5 in every iteration. Initially, $\mathscr{V}=\{0\}$ which is connected. Next, assuming there are $n$ agents in $\mathscr{V}$ and the graph they form is connected, we will 
prove that after adding the $(n+1)$ th agent, say $i$, the graph remains connected.

The addition of $i$ to $\mathscr{V}$ occurs at Step 5. There are two possible sequences for reaching this step: 1-2-4 and 1-2-3-4. At Step $2, \mathscr{U}_{i} \cap \mathscr{V} \neq \emptyset$ because of the property of $Q(\mathscr{G}(\mathbf{s}))$ in Lemma 4. It follows that before $i$ performs the procedure, there is at least one upstream agent in $\mathscr{V}$. In the 1-2-4 sequence, there exists some $m \in \mathscr{V} \cap \mathscr{U}_{i}$ such that $c\left(\hat{s}_{i}, s_{m}(t+\varepsilon)\right)=1$. Therefore, all agents in $\mathscr{V}$ including $i$ will be connected. In the 1-2-3-4 sequence, at Step 3, agent $i$ 's position is projected onto the connection ranges of all $v \in \mathscr{V} \cap \mathscr{U}_{i}$. It follows that the graph formed by agents in $\{\mathscr{V}, i\}$ is connected. Step 5 adds agents to $\mathscr{V}$ one by one until $\mathscr{V}=\mathscr{N}$, therefore, the graph $\mathscr{G}(\mathbf{s}(t+\varepsilon))$ is connected.

\section{References}

Bertsekas, D.P., 1995. Nonlinear Programming. Athena Scientific.

Bertsimas, D., Tsitsiklis, J.N., 1997. Introduction to linear optimization. volume 6. Athena Scientific Belmont, MA.

Breitenmoser, A., Schwager, M., Metzger, J.C., Siegwart, R., Rus, D., 2010. Voronoi coverage of non-convex environments with a group of networked robots, in: Proc. of the 2010 IEEE International Conference on Robotics and Automation, pp. 4982-4989.

Caicedo-Nuez, C., Zefran, M., 2008. A coverage algorithm for a class of non-convex regions, in: Proc. of the 47th IEEE Conf. on Decision and Control, pp. 4244-4249.

Caicedo-Nunez, C.H., Zefran, M., 2008. Performing coverage on nonconvex domains, in: Proc. of the 2008 IEEE Conf. on Control Applic., pp. 1019-1024.

Cao, Y., Yu, W., Ren, W., Chen, G., 2013. An overview of recent progress in the study of distributed multi-agent coordination. IEEE Transactions on Industrial Informatics 9, 427-438. doi:10.1109/TII.2012.2219061.

Cassandras, C., Lin, X., Ding, X., 2013. An optimal control approach to the multi-agent persistent monitoring problem. Automatic Control, IEEE Transactions on 58, 947961. doi:10.1109/TAC.2012.2225539.

Cassandras, C.G., Li, W., 2005. Sensor networks and cooperative control. European Journal of Control 11, 436-463.

Choi, J., Oh, S., Horowitz, R., 2009. Distributed learning and cooperative control for multi-agent systems. Automatica 45, $2802-2814$.

Cortes, J., Martinez, S., Karatas, T., Bullo, F., 2004. Coverage control for mobile sensing networks. IEEE Transactions on Robotics and Automation 20, 243-255. doi:10.1109/TRA.2004.824698.

Desai, J., Kumar, V., Ostrowski, J., 1999. Control of changes in formation for a team of mobile robots, in: Proc. of the IEEE International Conference on Robotics and Automation, pp. 1556-1561 vol.2.

Gusrialdi, A., Dirza, R., Hatanaka, T., Fujita, M., 2013. Improved distributed coverage control for robotic visual sensor network under limited energy storage. Int. J. Imaging Robot 10, 58-74.
Ji, M., Egerstedt, M.B., 2007. Distributed coordination control of multi-agent systems while preserving connectedness. IEEE Transactions on Robotics 23, 693-703.

Köppe, M., 2012. On the complexity of nonlinear mixedinteger optimization, in: Mixed Integer Nonlinear Programming. Springer, pp. 533-557.

Merris, R., 1994. Laplacian matrices of graphs: a survey. Linear algebra and its applications 197, 143-176.

Olfati-Saber, R., Murray, R., 2004. Consensus problems in networks of agents with switching topology and timedelays. IEEE Transactions on Automatic Control 49, 1520-1533. doi:10.1109/TAC.2004.834113.

Sakurama, K., Azuma, S.I., Sugie, T., 2015. Distributed controllers for multi-agent coordination via gradient-flow approach. Automatic Control, IEEE Transactions on 60, 1471-1485. doi:10.1109/TAC.2014.2374951.

Schouwenaars, T., De Moor, B., Feron, E., How, J., 2001. Mixed integer programming for multi-vehicle path planning, in: Control Conference (ECC), 2001 European, IEEE. pp. 2603-2608.

Schwager, M., Bullo, F., Skelly, D., Rus, D., 2008. A ladybug exploration strategy for distributed adaptive coverage control, in: Proc. of the IEEE International Conference on Robotics and Automation, pp. 2346-2353.

Schwager, M., Rus, D., Slotine, J.J., 2011. Unifying geometric, probabilistic, and potential field approaches to multirobot deployment. The International Journal of Robotics Research 30, 371-383.

Shamma, J., 2008. Cooperative Control of Distributed MultiAgent Systems. Wiley-Interscience, New York, NY, USA.

Sun, X., Cassandras, C., Gokbayrak, K., 2014. Escaping local optima in a class of multi-agent distributed optimization problems: A boosting function approach, in: Proc. of the IEEE 53rd Annual Conference on Decision and Control, pp. 3701-3706. doi:10.1109/CDC.2014.7039965.

Wang, J., Xin, M., 2013. Integrated optimal formation control of multiple unmanned aerial vehicles. IEEE Transactions on Control Systems Technology 21, 1731-1744. doi:10.1109/TCST.2012.2218815.

Yamaguchi, H., Arai, T., 1994. Distributed and autonomous control method for generating shape of multiple mobile robot group, in: Proc. of the IEEE International Conference on Intelligent Robots and Systems, pp. 800-807 vol.2. doi:10.1109/IROS.1994.407547.

Zhong, M., Cassandras, C., 2011. Distributed coverage control and data collection with mobile sensor networks. IEEE Transactions on Automatic Control 56, 2445-2455. doi:10.1109/TAC.2011.2163860. 